\title{
Ansiedad como predictor de la conducta impulsiva en niños con sobrepeso y obesidad
}

\section{Anxiety as a predictor of impulsive behavior in overweight and obese children}

\author{
Javier Nahúm Alfaro Belmont, Patricia Romero Sánchez \\ y Ariel Vite Sierra
}

\author{
Universidad Nacional Autónoma de México ${ }^{1}$
}

Autor para correspondencia: Javier N. Alfaro Belmont, janvierstyle@comunidad.unam.mx.

\section{RESUMEN}

\begin{abstract}
El objetivo de esta investigación fue evaluar la relación entre la ansiedad y la impulsividad en infantes con sobrepeso y obesidad mediante la escala de ansiedad para niños SCAS y el sistema de evaluación de conducta impulsiva, que mide el patrón impulsivo a través de cuatro dimensiones del reforzador. Cabe mencionar que el estudio se realizó a lo largo de cinco sesiones para obtener el promedio de elección. Participaron 58 niños de tercero, cuarto y quinto grados de dos escuelas primarias ubicadas en el sur de la ciudad de México. Se llevó a cabo un análisis de correlación de Pearson en diferentes subgrupos (en cuanto a género, condición de sobrepeso u obesidad y grado escolar) entre las subescalas y el puntaje total del SCAS respecto a las dimensiones asociadas a la elección, así como un análisis de regresión lineal para observar la varianza explicada tomando la ansiedad como predictor y la impulsividad como variable dependiente. Los resultados obtenidos muestran que los niveles de ansiedad más altos se observaron en los niños con obesidad o sobrepeso. También se describe una relación positiva moderada entre la ansiedad y la impulsividad en diferentes subgrupos. En cuanto a los factores de predicción, el grupo de tercer grado mostró una mayor varianza explicada por los niveles de ansiedad en la elección inmediata de alimentos, aunque se encontraron más modelos que explican la elección en los niños de quinto. En conclusión, se halló una relación entre las variables de ansiedad e impulsividad, siendo la ansiedad por separación, el miedo al daño físico y la fobia social los factores que mejor predicen las dimensiones de impulsividad. Se considera pertinente el estudio de la interacción de ambas variables al llevar a cabo estrategias de prevención e intervención dirigidas a esta población.
\end{abstract}

Palabras clave: Obesidad infantil; Impulsividad; Ansiedad; Elección; Escuelas primarias.

\begin{abstract}
The objective of the present study was to evaluate the relationship between anxiety and impulsivity in overweight and obese elementary schoolchildren. Measurement included the anxiety scale for children SCAS composed by seven subscales, and the impulsive behavior assessment system (SECI) that measures impulsive patterns across four dimensions of food as a reinforcer. Data were collected over five sessions to obtain the average selected food choice. A total of 58 children from $3 \mathrm{rd}$, 4 th and 5th grades from two elementary schools located in southern Mexico City participated. Pearson correlation analyses were conducted for the subgroups: gender, overweight or obesity status, and school grade. The subscales and the total SCAS score explored the dimensions associated with food choice. Also linear regression analyses were performed in order to identify the variance explained
\end{abstract}

\footnotetext{
${ }^{1}$ Facultad de Psicología, Av. Universidad 3004, Col. Copilco Universidad, Álvaro Obregón, 04510 Ciudad de México, México, correos electró-
} nicos: janvierstyle@comunidad.unam.mx,pattyro74@comunidad.unam.mx y avite@unam.mx. 
with anxiety as predictor and impulsivity as dependent variable. The results show that the highest anxiety levels occurred in children who were obese or overweight, as well as a positive, moderate relationship between anxiety and impulsivity in different subgroups. As for predictive factors, the 3 rd grade group showed greater variance explained by anxiety levels for the immediate choice of food. Other models explained the food choice in 5 th graders. We concluded that there is a definite relationship between anxiety and impulsivity, with the strongest predictive factors being impulsivity, separation anxiety, fear of physical damage and social phobia. Thus the study of the interaction of these variables when designing prevention and intervention strategies aimed at the health of children is highly relevant.

Keywords: Childhood obesity; Impulsivity; Anxiety; Choice; Elementary school

Recibido: 22/10/2019

Aceptado: 30/05/2020

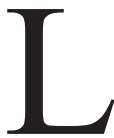

a obesidad en México ha aumentado en los últimos años. Se ha estimado que en 2012 había 20.5 millones de adultos mayores de 18 años, cifra que ascendió a 24.3 millones en 2016 (Organización de las Naciones Unidas para la Alimentación y la Agricultura [FAO], 2018). Respecto a la obesidad infantil, un reporte realizado en 2020 por Shamah y Rivera basado en la Encuesta Nacional de Salud y Nutrición (ENSANUT) mostró que la prevalencia combinada de sobrepeso y obesidad es de $35.6 \%$ en niños de 5 a 11 años. Además, la Organización para la Cooperación y Desarrollo Económicos (OCDE) (2017) llevó a cabo una actualización respecto a la obesidad infantil, indicando que uno de cada seis niños presenta este problema, y se estima que para 2030 se eleve a cuatro de cada diez.

El sobrepeso y obesidad se han definido como una acumulación anormal o excesiva de grasa que es perjudicial para la salud. Para operacionalizar el grado de afectación o severidad, la Organización Mundial de la Salud (OMS) actualizó sus datos en 2006 con base en los patrones de crecimiento de niños y adolescentes de entre $5 \mathrm{y}$ 19 años, donde se pueden obtener los promedios y desviaciones estándar según el sexo y edad en años y meses; asimismo, la OMS ha establecido tablas fijas para adultos mayores a 18 años (OMS, 2018). Estos parámetros de la edad exacta se toman en cuenta para detectar un problema con el peso.

El índice de masa corporal (IMC en lo sucesivo) es el indicador más utilizado para identificar los problemas de peso. Se calcula a partir de la fórmula: peso $(\mathrm{kg}) / \mathrm{talla}\left(\mathrm{m}^{2}\right)$. Según los parámetros de desarrollo entre los 5 y 19 años, se considera que una persona presenta sobrepeso si su IMC se ubica por arriba de una y hasta dos desviaciones estándar respecto de la media esperada para la edad, mientras que aquellos con una puntuación por arriba de dos desviaciones respecto de la media son considerados como personas con obesidad (Rivera et al., 2018).

El sobrepeso y la obesidad son una condición de salud que se asocia con otros problemas, como enfermedades cardiovasculares, trastornos del aparato locomotor (osteoartritis), cáncer, dificultades respiratorias, fracturas y diabetes. Por ello es imprescindible e importante el estudio de este problema de salud, así como de las variables relacionadas con su prevención e intervención.

En México, el patrón alimenticio esta circunscrito a diferentes factores distales, como la calidad de los productos elaborados y procesados, los sustitutos de azucares o vitaminas sin una adecuada regulación de su control de calidad, los alimentos "chatarra" de precio accesible que favorecen el desarrollo de comportamientos alimenticios implicados en los problemas de peso (Rivera et al., 2018). Estas condiciones, en conjunto con el gradiente de desigualdad social que restringe la accesibilidad a los servicios urbanos, convierten a muchos sectores de la población infantil en grupos en riesgo de padecer problemas de salud, como la obesidad y la desnutrición (Luna et al., 2020; Torres, Rodríguez y Silva, 2020).

Además de lo anterior, la preferencia infantil por alimentos poco saludables conlleva que entre los niños de 5 a 11 años (edad escolar), más de la mitad reporte el consumo de bebidas endulzadas, botanas y carnes procesadas, por encima de otros rangos de edad. Si se considera que en México están registrados 22 millones de niños de entre 0 y 10 años con esas características, y que la prefe- 
rencia de más de la mitad de ellos se inclina por este tipo de alimentos; luego entonces, existe un importante factor de riesgo para que gran parte de la población infantil desarrolle problemas de sobrepeso y obesidad (Torres y Rojas, 2018).

En el estudio del comportamiento alimenticio bajo la perspectiva de la psicología conductual se ha identificado que los patrones conductuales descritos de manera general como autocontrol e impulsividad se asocian con el consumo de grandes e inmediatas cantidades de comida con un mínimo esfuerzo debido a su bajo costo o proximidad, lo que se relaciona estrechamente con la elección de la misma.

La sensibilidad a la recompensa -es decir, conductas dirigidas a la obtención de refuerzos tales como dinero, poder, sexo y búsqueda de sensaciones (Pascual, Pascual, Redondo y Pérez, 2014)puede configurar la elección por determinados alimentos (salados, dulces y grasosos), ya que tienen un valor gratificante mayor respecto a aquellos con bajo contenido calórico. Las personas con sobrepeso y obesidad, en comparación con aquellas con normopeso, eligen comida chatarra e inmediata (Davis et al., 2007) y muestran una tendencia a actuar de manera espontánea y a tomar decisiones precipitadas (Ryden et al., 2003). En este sentido, el estudio de la toma de decisiones en su dimensión conductual (la impulsividad) podría resultar útil para evaluar si un patrón de comportamiento está regulado por diferentes variables asociadas a la elección de comida saludable o chatarra (Vite, Negrete y Cavita, 2020).

Se ha definido la impulsividad como la reacción rápida, no planeada, ante estímulos internos o externos sin valorar las consecuencias (Meule, 2013). Diferentes teorías clásicas explican su adquisición y desarrollo (Bandura, 1978; Baumrind, 1971; Patterson y Wells, 1982), así como el control ejecutivo asociado con la toma de decisiones (Logan, 1985).

Se han llevado a cabo estudios en los que se emplean autoinformes y pruebas psicométricos (Iribarren, Jiménez, García y Rubio, 2011; Plutchik, Van Praag, Conte y Picard, 1989; Salvo y Castro, 2013), así como pruebas conductuales, afines a evaluaciones situacionales en vivo para la toma de decisiones en jóvenes obesos (Chamberlain, Derbyshire, Leppink y Grant, 2015), hallándose que las personas con obesidad muestran tasas altas de conductas desadaptativas, como la elección inmediata y las pérdidas monetarias; a través de un análisis multivariado, se ha visto que el sexo femenino y un bajo control inhibitorio predicen significativamente la obesidad.

Por su parte, Fields, Sabet y Reynolds (2013) reportaron que los preadolescentes con sobrepeso y obesidad eran más impulsivos en cuanto a las tareas de atención que se les presentaban (esto es, tardaban menos tiempo en responderlas) y eran más sensibles al descuento temporal (preferir recompensas inmediatas inferiores en lugar de recompensas superiores a futuro).

Vite et al. (2020), en un estudio realizado en niños mexicanos, encontraron que las elecciones de niños con sobrepeso y obesidad se veían influidas por la inmediatez del reforzador (obtener comida inmediatamente) y esperar poco tiempo para pagar (esforzarse) el alimento.

Considerando que la impulsividad es un fenómeno multifactorial que abarca desde la serie de elecciones planeadas o no planeadas ante cualquier decisión que se tome (p. e., consumir determinado tipo de comida, a qué hora, con qué frecuencia y cantidad), hasta condicionantes tales como la disponibilidad, el valor de gratificación, el esfuerzo requerido para obtenerla y el tiempo en que se obtendrá (Neef, Bicard y Endo, 2001), es posible extender su estudio al comportamiento de elección hacia alimentos chatarra, y tener así evidencia de cómo los mismos se asocian con los problemas de sobrepeso y la obesidad, en virtud de que estos son resultado en gran medida de la dieta elegida, que incluye alimentos altamente calóricos por su valor gratificante.

En el terreno de la impulsividad hay investigaciones que la relacionan con variables psicológicas como la ansiedad. En la literatura es factible encontrar esas asociaciones en la población adolescente (Corbí y Pérez, 2011) con la violencia familiar (Negrete y Vite, 2011) o las adicciones (Gran y Nieto, 2012; Gutiérrez, Valladolid y De Fonseca, 2013). Sin embargo, poco se conoce respecto de cómo se relaciona el comportamiento im- 
pulsivo en situaciones de elección con los niveles de ansiedad mostrados por la población infantil o adolescente con sobrepeso y obesidad.

La ansiedad psicológica clínicamente significativa puede fomentar el aumento de peso y la obesidad, lo que conduce a sufrir problemas psicosociales, como los conductuales de externalización (impulsividad y déficit de atención e hiperactividad), internalización (depresión y ansiedad) y un comportamiento alimentario incontrolado (Puder y Munsch, 2010). Se estima que cerca de 260 millones personas en el mundo padecen algún trastorno de ansiedad, la cual es considerada como la sexta causa de enfermedad y discapacidad en niños (OMS, 2019). En México, se estima que $14 \%$ de la población los padece, siendo los problemas de salud mental más prevalentes en el país. También se ha reportado que en la ciudad de México ha habido un considerable incremento en el número de consultas externas y de hospitalizaciones por sintomatología ansiosa y estrés en la población de entre 0 y 18 años (Márquez, Arroyo, Granados y Ángeles (2017).

Los niveles de alta ansiedad se han relacionado con el control inhibitorio y la sensibilidad a la recompensa (elección de recompensas inmediatas), y se consideran factores psicológicos implicados en el desarrollo y mantenimiento de la obesidad pediátrica (Goldschmidt, Smith, Lavender, Engel y Haedt-Matt, 2019). Si lo anterior es correcto, entonces los paradigmas de elección sirven para evaluar cualquier problemática relacionada con la toma de decisiones derivada de patrones ansiosos (Pereira y Chehter, 2011).

En una revisión sistemática llevada a cabo por Giel, Teufel, Junne, Zipfel y Schag (2017) de los resultados obtenidos en veinte estudios con diversos diseños experimentales, se describe que la elección de comida inmediata y el atracón pueden considerarse como un fenotipo en el espectro de la obesidad. En ellos se utilizaron tareas clásicas de elección entre imágenes de alimentos, hallándose que la sensibilidad a la recompensa se asociaba a la inmediatez y disponibilidad de los alimentos. Específicamente en los niños, se vincula el sobrepeso con bajos niveles de inhibición a la respuesta (por ejemplo, elegir más alimentos en el menor tiempo), lo cual se ha asociado con la conducta impulsiva (Nederkoorn, Coelho, Guerrieri, Houben y Jansen, 2012), aunque la evidencia sugiere considerar más estudios que incorporen la ansiedad como una variable implicada en la toma de decisiones (Goldschmidt et al., 2019).

Los paradigmas más utilizados para analizar la impulsividad son aquellos en los que los participantes deben elegir, en programas concurrentes, una recompensa pequeña inmediata, o mayor pero demorada (Chamberlain et al., 2015; Neef et al., 2001; Sánchez, Giraldo y Quiroz, 2013). Con base en el análisis conductual aplicado, la utilización de técnicas de evaluación conductual resultaría eficaz bajo el principio del establecimiento de una línea base para obtener un patrón de elección en cuando menos 3 momentos diferentes, con el objetivo de establecer un patrón de comportamiento impulsivo en más de una ocasión.

En esta línea, Neef et al. (2001) proponen una serie de dimensiones relacionadas con la elección impulsiva en la que se señalan cuatro categorías principales: inmediatez, esfuerzo, calidad y programa de reforzamiento. Los citados autores señalan que la interacción de estas cuatro dimensiones al momento de elegir indican cuál de ellos tiene más peso para cada individuo. Por ejemplo, algunos niños eligen lo inmediato, aunque se esforzarán más para obtenerlo, y en otras ocasiones querrán obtener el reforzador con poco esfuerzo, aunque deban esperar más tiempo. De esta manera, si un niño elige lo inmediato con poco esfuerzo y espera poco tiempo para lograr la recompensa que obtendrá, se puede decir que muestra un patrón de comportamiento impulsivo.

Los primeros planteamientos que hicieron los investigadores citados fueron respecto al complejo de elección de niños con problemas de déficit de atención e hiperactividad. Con base en ello, desarrollaron un software en el que colocaban dos pantallas concurrentes asociadas con un reforzador, cuya entrega dependía de que el niño respondiera una operación matemática básica relacionada con un programa de reforzamiento, vinculado al tiempo en que tenía que esperar para responder tal operación. A partir de este arreglo, midieron las dimensiones de elección impulsiva: Calidad de 
reforzador, asociado a la preferencia de objetos, imágenes, comida saludable o chatarra; Inmediatez, tiempo de espera en que se entrega el reforzador; por ejemplo, un minuto o mañana; Esfuerzo, relativo al requisito alto o bajo de respuesta para obtener ese reforzador, y Programa de reforzamiento, relacionado al tiempo que debe esperar el participante para acceder al reforzador (por ejemplo, 30, 60 o 90 segundos).

Con base en lo antes descrito, se han generado tareas que incorporan las cuatro dimensiones para establecer los patrones de comportamiento impulsivo, como el papel que en la impulsividad de los adolescentes tienen la violencia familiar (Negrete y Vite, 2011), los problemas de conducta (Caporal, Moysen y Vite, 2015), la obesidad infantil (Cavita y Sánchez, 2012; Vite et al., 2020; Romero y Zúñiga, 2016) y el déficit de atención con hiperactividad (Neef et al., 2005).

Se ha considerado la impulsividad como un rasgo común entre la elección de comida chatarra, inmediata y con bajo esfuerzo, patrón de comportamiento que se asocia a los problemas de sobrepeso y obesidad. Parece necesario indagar más en la relación entre estas variables, así como identificar si tales asociaciones tienen rasgos similares al comparar los casos de sobrepeso -antesala de problemas crónico-degenerativos como el cáncer, la diabetes o la hipertensión-y la obesidad. Así, se abre el campo para trabajar con la impulsividad mediante un programa de intervención o prevención, ya que la modificación de la conducta impulsiva en situaciones de elección puede ser un eje principal para el cambio de hábitos de alimentación, elección de tipo de alimentos y realización de actividad física, los que pueden operar para mantener un comportamiento saludable (Sánchez et al., 2013).

Con base en lo anterior, es posible observar que el campo de conocimiento donde se relacionan variables como la impulsividad y la ansiedad en niños con sobrepeso y obesidad es limitado cuando se utiliza un paradigma de elección, por lo que un análisis que pretenda describir en qué grado están asociadas las variables puede ser el primer paso para fundamentar intervenciones basadas en trabajo con la variable de elección y mediadas por cuestiones emocionales o cognitivas (depresión, ansiedad, miedo, frustración, etc.).

Debido a que la elección de alimentos alude a una amplia gama de factores sociales, como la familia, la cultura o la disponibilidad, y psicológicos como la recompensa inmediata en menor tiempo, la elección de productos que dan la sensación de dejar a la persona satisfecha más rápidamente, la integración de modelos enfocados en la elección podría ser de utilidad para el manejo de los hábitos saludables.

Cabe mencionar que el papel de las variables sociales en las elecciones impulsivas, así como el de la ansiedad en los patrones de comportamiento alimenticio, son de vital importancia para llevar a cabo un diagnóstico preciso y generar así estrategias de prevención e intervención con fundamento en las recomendaciones hechas por instancias nacionales e internacionales (Márquez et al., 2017; OCDE, 2017; OMS, 2019).

Es escaso el estudio de los factores relacionados con el sexo, la edad y otras variables sociodemográficas en la ansiedad como mediadora entre la impulsividad y la conducta alimentaria. Incorporar variables como el grado escolar y el sexo como factores implicados en la modificación de hábitos saludables, entre ellos la alimentación del niño, podría aportar evidencias sobre la forma en que se relacionan las variables de interés y haría posible el desarrollo de intervenciones centradas en el comportamiento impulsivo relacionado con la ansiedad.

Por tanto, el objetivo del presente estudio fue observar la relación entre las dimensiones de impulsividad y las subescalas de ansiedad en niños con problemas de sobrepeso y obesidad. Específicamente, se pretendió describir los puntajes promedio de las variables de ansiedad y las dimensiones de elección en tales niños a partir de su sexo y grado escolar, así como también identificar si los indicadores de ansiedad son predictores de las dimensiones de elección, y si estas predicciones difieren de acuerdo con la condición de salud (sobrepeso u obesidad), el sexo y el grado de escolaridad de los menores. 


\section{MÉTODO}

\section{Participantes}

Participaron en el estudio 58 niños, de los cuales 26 fueron mujeres, con una edad media de 9 años $($ D.E. $=.83)$, seleccionados de manera intencional en dos escuelas primarias públicas ubicadas al sur de la ciudad de México.

\section{Materiales e instrumentos}

Escala de Ansiedad para Niños de Spence (Spence Children's Anxiety Scale, SCAS) (Spence, 1997).

Se utilizó la versión en español adaptada por Hernández et al. (2010) para niños mexicanos. Está compuesta por 38 ítems referidos a síntomas de ansiedad, con cuatro opciones de respuesta tipo Likert: de "nunca" (0) a "siempre" (3). Su consistencia interna, medida por el coeficiente alfa de Cronbach, es de .88, y de .81 para el factor Ataque de pánico/agorafobia; de .74 para Ansiedad de separación, de .71 para el de Fobia social, de .75 para el factor Miedo al daño físico, de .77 para el factor Trastorno obsesivo-compulsivo, y finalmente, de .72 para el de Ansiedad generalizada. La escala ofrece ciertas ventajas, como sensibilidad a los cambios ejercidos por el tratamiento, capacidad para discriminar entre muestras clínicas y población general, aplicación transcultural y, muy especialmente, apego a la clasificación diagnóstica de los trastornos de ansiedad más comunes del DSM.

\section{Sistema de Evaluación de Conducta Impulsiva} (SECI) (Negrete, Cuevas y Vite, 2009).

La evaluación de la conducta impulsiva se llevó a cabo por medio de este instrumento, el cual permite evaluar la conducta impulsiva de un individuo basándose en las elecciones hechas con dos opciones de respuesta (elección de problemas aritméticos), presentadas concurrentemente y cuyas características varían de acuerdo a las dimensiones del reforzador (en este caso, música en formato mp3). La evaluación de la conducta impulsiva por medio de este software se fundamentó en la línea de investigación de Neef y sus colaboradores (Neef y Lutz, 2001; Neef et al., 2005; Neef, Mace y Shade, 1993).
Las dimensiones del reforzador operadas por el software fueron las siguientes:

Inmediatez (I). La inmediatez del reforzamiento hace referencia a la condición de elección entre un reforzador entregado inmediatamente (al finalizar la sesión), contra otro reforzador, pero por el cual se debe esperar un determinado tiempo para ser entregado (por ejemplo, al día siguiente).

Calidad (C). La calidad de un reforzador alude a la preferencia relativa de un reforzador sobre otro. En este experimento, un reforzador era de mayor o menor calidad dependiendo del lugar que ocupase en el muestreo de reforzadores. Los reforzadores disponibles dentro del software correspondían a alimentos chatarra (calidad baja) y comida saludable (calidad alta).

Programa de reforzamiento ( $R$ ). Se refiere al programa concurrente de reforzamiento en efecto sobre un respectivo conjunto de problemas aritméticos. Para un programa de reforzamiento alto se empleó un programa de reforzamiento intervalo variable de 5 segundos (IV-5s) y para un programa bajo un IV-60s.

Esfuerzo de la respuesta (E). Hace referencia a la dificultad relativa con la que un problema aritmético puede ser resuelto. En este experimento, el esfuerzo fue la dificultad del problema aritmético que estaba en función del número de sumandos que son acarreados a una posición superior de una operación aritmética. Para corroborar que estos rangos de dificultad fueron percibidos de la misma manera por los niños, se realizó una validación por jueces, quienes ajustaban las sumas para cada niño dependiendo de su edad.

\section{Escenario}

La aplicación del SCAS y el programa SECI se llevó a cabo en el salón de cómputo de ambas escuelas, un espacio cerrado de 4 x $4 \mathrm{~m}$ con adecuada iluminación, ventilación y libre de distractores y ruido.

\section{Variables}

Ansiedad: Evaluada mediante la escala de ansiedad para niños, en la que los puntajes altos corresponden a mayor ansiedad. 
Impulsividad: La relación existente entre la frecuencia de respuestas que impliquen la elección de comida menos saludable, con menos esfuerzo (sumas más fáciles), escasa demora y programa de reforzamiento alto para contestar una operación matemática. Con ayuda del software se obtiene un patrón de elección a lo largo de cinco sesiones para lograr la estabilidad de la respuesta, promediándose después.

\section{PROCEDIMIENTO}

Una vez contactadas las escuelas y de haber obtenido de ellas los permisos correspondientes para la aplicación, así como el consentimiento de los padres y los niños, se trabajó en el aula de cómputo, donde se les pedía que, en una sesión de 30 minutos, llenaran el instrumento.

Se tuvo especial cuidado en que la aplicación estuviera fundada en los cuidados instrumentales, metodológicos, normas de conducta, supervisión y conducción de la investigación indicados en los artículos correspondientes (Artículos 1, 2, 8, 16, 47,48 ), y sobre el consentimiento informado (Artículos 118-131) del Código Ético del Psicólogo de la Sociedad Mexicana de Psicología (2010).

Respecto a la instrumentación del software, este se aplicó una vez a la semana durante cinco sesiones de 40 minutos cada una, aproximadamente. Al principio de cada sesión se evaluaba la preferencia relativa del niño bajo un procedimiento de muestreo de reforzadores (veinte tipos de alimentos chatarra y veinte de comida saludable); así, el programa discriminaba los tres tipos más preferidos (reforzador alto) y los tres tipos menos preferidos (reforzador bajo) para completar la dimensión Calidad (C).

Después del muestreo, en un monitor de computadora se le presentaba al niño una pantalla de elección en la que debía seleccionar de entre dos problemas aritméticos, aquel que prefiriera, de acuerdo con los niveles de las dimensiones del reforzador. Esta pantalla de elección mostraba un problema aritmético del lado izquierdo y uno del lado derecho, cada uno de los cuales tenía como fondo un color asociado al programa de reforzamiento (R), el que podía ser en color amarillo (IV
5 segundos), blanco (IV 30 segundos) o rojo (IV 60 segundos). En la parte inferior de cada problema se indicaba cuándo podría obtener virtualmente ese alimento, lo que podía ser de manera inmediata (I, al final de la sesión) o demorada (D, al día siguiente).

Una vez que el niño escogía el problema aritmético, la pantalla de elección desaparecía para mostrar únicamente el ejercicio seleccionado. Después de haber transcurrido el tiempo asociado al programa de reforzamiento, aparecían las opciones de respuesta en un formato de opción múltiple. Si el niño contestaba correctamente, una ventana así lo indicaba, y el punto era asignado al contador correspondiente al tipo de comida elegida; después se mostraba una nueva pantalla de elección con nuevos problemas aritméticos y una nueva oportunidad de elección ${ }^{2}$.

Evaluación de la conducta impulsiva. A lo largo de las cinco sesiones de evaluación, las cuatro dimensiones del reforzador fueron puestas en competencia directa a través del "Programa de reforzamiento vs. Inmediatez", "Calidad vs. Inmediatez" e "Inmediatez vs. Esfuerzo". De este modo, en una pantalla de elección competían dos dimensiones, mientras que las otras dos permanecían constantes. La evaluación terminaba hasta que el niño completaba 30 pantallas de elección o transcurrían 30 minutos de evaluación.

Los datos obtenidos en cuanto a la elección se analizaron en una base de datos y se obtuvieron los promedios de las cinco sesiones de evaluación para observar el grado relativo de preferencia por cada dimensión; es decir, cuál de las cuatro dimensiones resultaba influir más a lo largo de cinco sesiones $y$, en consecuencia, mostraba un perfil para, a su vez, relacionarla con la ansiedad.

En cuanto al instrumento, se obtuvieron los resultados totales y por subescalas. Luego, se vaciaron en una base de datos los promedios de las dimensiones de impulsividad y las subescalas de ansiedad para realizar un análisis descriptivo de los participantes y proceder a efectuar un análisis de correlación de Spearman. Finalmente, se analizaron los resultados mediante el programa SPSS, v. 22.

${ }^{2}$ Para una mejor comprensión de las pantallas de competencias de las dimensiones en el Sistema de Evaluación de la Conducta Impulsiva (SECI), véase Negrete et al., 2009). 


\section{RESULTADOS}

En la Tabla 1 se muestran los datos descriptivos de los promedios en niveles de ansiedad, las subescalas de la misma y las dimensiones asociadas a las elecciones agrupadas, según la condición de sobre- peso y el grado escolar. En los 58 participantes se obtuvo una media de ansiedad de 59.5 (D.E. $=19.3$ ), teniendo en cuenta que la escala de ansiedad se clasifica en tres niveles: alto (78-114 pts.), medio (39-77 pts.) o bajo (0-38 pts.).

Tabla 1. Media y desviación estándar en los subgrupos de niños con sobrepeso y obesidad.

\begin{tabular}{|c|c|c|c|c|c|c|c|c|c|}
\hline \multirow{3}{*}{ Subgrupo } & & \multicolumn{4}{|c|}{ Sobrepeso $(n=27)$} & \multicolumn{4}{|c|}{ Obesidad $(n=31)$} \\
\hline & & \multicolumn{2}{|c|}{ Niñas $(n=14)$} & \multicolumn{2}{|c|}{ Niños $(n=13)$} & \multicolumn{2}{|c|}{ Niñas $(n=12)$} & \multicolumn{2}{|c|}{ Niños $(n=19)$} \\
\hline & & M & D.E. & $\mathbf{M}$ & D.E. & $\mathbf{M}$ & D.E. & $\mathbf{M}$ & D.E. \\
\hline \multirow{11}{*}{$\begin{array}{c}3^{\circ} \\
(n=15)\end{array}$} & Ansiedad generalizada & 6.67 & 1.15 & 13.00 & 3.46 & 12.00 & 4.24 & 13.40 & 2.30 \\
\hline & Miedo al daño físico & 12.33 & 4.93 & 13.00 & 4.58 & 12.50 & 6.40 & 12.60 & 3.78 \\
\hline & Ansiedad por separación & 6.33 & 4.93 & 7.00 & 4.58 & 12.25 & 8.50 & 10.60 & 4.98 \\
\hline & Fobia social & 9.67 & 1.15 & 13.67 & 2.31 & 13.00 & 2.83 & 14.00 & 1.41 \\
\hline & Pánico con agorafobia & 10.33 & 7.51 & 19.00 & 8.66 & 11.50 & 10.34 & 15.20 & 6.30 \\
\hline & Trastorno obsesivo compulsivo & 3.33 & 5.77 & 13.00 & 3.46 & 9.50 & 4.80 & 9.60 & 4.83 \\
\hline & Ansiedad total & 48.67 & 14.74 & 78.67 & 24.83 & 70.75 & 27.35 & 75.40 & 17.40 \\
\hline & ${ }^{\circ}$ Calidad & 19.62 & 11.79 & 22.95 & 13.15 & 18.63 & 9.87 & 24.44 & 6.35 \\
\hline & ${ }^{\circ}$ Inmediatez & 7.74 & 4.63 & 7.33 & 7.28 & 11.49 & 5.15 & 12.19 & 7.24 \\
\hline & ${ }^{\circ}$ Esfuerzo & 39.31 & 4.22 & 36.20 & 16.09 & 40.81 & 8.54 & 33.48 & 19.91 \\
\hline & ${ }^{\circ}$ Programa de reforzamiento & 38.32 & 11.30 & 38.19 & 4.98 & 33.62 & 7.11 & 33.64 & 10.04 \\
\hline \multirow{11}{*}{$\begin{array}{c}4^{\circ} \\
(n=18)\end{array}$} & Ansiedad generalizada & 4.00 & 4.58 & 8.75 & 6.34 & 9.40 & 4.83 & 9.50 & 5.82 \\
\hline & Miedo al daño físico & 4.33 & 4.51 & 8.50 & 5.00 & 11.00 & 4.69 & 10.00 & 4.52 \\
\hline & Ansiedad por separación & 2.00 & 2.00 & 8.75 & 2.06 & 2.80 & 4.09 & 10.67 & 5.57 \\
\hline & Fobia social & 7.67 & 3.06 & 10.75 & 4.79 & 11.40 & 3.29 & 11.33 & 3.88 \\
\hline & Pánico con agorafobia & 8.00 & 7.00 & 9.50 & 3.11 & 8.80 & 7.26 & 14.50 & 6.95 \\
\hline & Trastorno obsesivo compulsivo & 2.67 & 2.52 & 7.25 & 6.40 & 6.00 & 2.92 & 7.00 & 4.69 \\
\hline & Ansiedad total & 28.67 & 2.08 & 53.50 & 21.21 & 49.40 & 13.74 & 63.00 & 13.02 \\
\hline & ${ }^{\circ}$ Calidad & 19.52 & 19.04 & 26.68 & 16.13 & 21.56 & 15.42 & 17.46 & 11.78 \\
\hline & ${ }^{\circ}$ Inmediatez & 11.01 & 9.90 & 12.51 & 8.46 & 8.51 & 3.03 & 13.37 & 7.89 \\
\hline & ${ }^{\circ}$ Esfuerzo & 33.84 & 7.54 & 21.78 & 8.21 & 39.62 & 12.69 & 37.06 & 10.47 \\
\hline & ${ }^{\circ}$ Programa de reforzamiento & 39.69 & 6.58 & 43.58 & 4.61 & 34.07 & 15.08 & 36.41 & 8.32 \\
\hline \multirow{11}{*}{$\begin{array}{c}5^{\circ} \\
(\mathrm{n}=25)\end{array}$} & Ansiedad generalizada & 9.88 & 5.17 & 10.83 & 4.36 & 7.67 & 4.73 & 8.88 & 4.02 \\
\hline & Miedo al daño físico & 9.38 & 5.83 & 11.83 & 1.94 & 13.00 & 2.65 & 10.75 & 4.43 \\
\hline & Ansiedad por separación & 4.63 & 1.85 & 6.83 & 2.71 & 5.33 & 6.81 & 6.50 & 5.81 \\
\hline & Fobia social & 11.50 & 3.63 & 12.33 & 2.73 & 10.33 & 3.21 & 11.00 & 2.56 \\
\hline & Pánico con agorafobia & 14.63 & 6.09 & 15.00 & 2.53 & 18.33 & 9.61 & 15.00 & 7.98 \\
\hline & Trastorno obsesivo compulsivo & 5.50 & 4.90 & 6.67 & 3.72 & 8.67 & 9.02 & 6.38 & 3.89 \\
\hline & Ansiedad total & 55.50 & 17.00 & 63.50 & 9.79 & 63.33 & 25.72 & 58.50 & 17.49 \\
\hline & ${ }^{\circ}$ Calidad & 19.24 & 15.54 & 23.23 & 11.25 & 22.11 & 10.74 & 20.74 & 13.79 \\
\hline & ${ }^{\circ}$ Inmediatez & 17.90 & 13.64 & 16.53 & 7.72 & 10.34 & 5.55 & 7.90 & 3.46 \\
\hline & ${ }^{\circ}$ Esfuerzo & 24.74 & 14.86 & 25.98 & 11.34 & 47.27 & 6.31 & 41.20 & 11.72 \\
\hline & 'Programa de reforzamiento & 42.35 & 9.88 & 38.47 & 12.46 & 24.14 & 19.87 & 33.42 & 10.01 \\
\hline
\end{tabular}


Se puede apreciar que las medias son similares en los diferentes subgrupos respecto a las subescalas de ansiedad. En cuanto a las dimensiones de impulsividad, los puntajes promedio son igualmnente similares en los diferentes subgrupos, y en relación a la ansiedad total, los niños en general puntúan más alto que las niñas en 3ro. y 4to. grados.

En referencia a las dimensiones de la impulsividad, los puntajes también fueron similares.
Cabe resaltar que en 5to. año la dimensión con mayor puntuación promedio fue $\mathrm{E}$ en los niños y niñas con obesidad.

Luego se llevó a cabo un análisis de correlación de Pearson en diferentes subgrupos y condiciones para identificar el grado de asociación entre las distintas dimensiones de ansiedad e impulsividad, cuyos resultados se muestran en la Tabla 2.

Tabla 2. Análisis de correlación de Pearson para las dimensiones de impulsividad y las subescalas de ansiedad significativas a $p \leq .05$.

\begin{tabular}{|c|c|c|c|c|}
\hline General $(n=58)$ & Calidad & Inmediatez & Esfuerzo & P. R. \\
\hline IMC & .005 & -.238 & $.298 *$ & -.242 \\
\hline Ansiedad generalizada & $.269 *$ & -.027 & -.201 & -.032 \\
\hline Miedo al daño físico & $.273 *$ & .028 & -.203 & -.089 \\
\hline Fobia social & $.263 *$ & -.022 & -.193 & -.040 \\
\hline Niñas $(\mathrm{n}=\mathbf{2 6})$ & Calidad & Inmediatez & Esfuerzo & P. R. \\
\hline Ansiedad generalizada & $.409 *$ & -.069 & $-.431 *$ & .093 \\
\hline Miedo al daño físico & $.414 *$ & .042 & -.229 & -.254 \\
\hline Fobia social & $.408 *$ & -.047 & $-.437^{*}$ & .084 \\
\hline Trastorno obsesivo compulsivo & .187 & .075 & .116 & $-.412 *$ \\
\hline Obesidad $(n=31)$ & Calidad & Inmediatez & Esfuerzo & P. R. \\
\hline Ansiedad por separación & -.105 & $.426 *$ & -.023 & -.093 \\
\hline $3^{\circ}(n=15)$ & Calidad & Inmediatez & Esfuerzo & P. R. \\
\hline Ansiedad por separación & -.118 & $.693 * *$ & -.103 & -.256 \\
\hline Ansiedad total & -.109 & $.526 *$ & -.093 & -.189 \\
\hline $5^{\circ}(n=25)$ & Calidad & Inmediatez & Esfuerzo & P. R. \\
\hline IMC & .001 & $-.523 * *$ & $.603 * *$ & -.346 \\
\hline Ansiedad generalizada & .156 & .078 & $-.397 *$ & .258 \\
\hline Fobia social & .170 & .094 & $-.414 *$ & .251 \\
\hline Pánico con agorafobia & $.529 * *$ & .169 & -.244 & $-.397 *$ \\
\hline Ansiedad total & $.433 *$ & .162 & -.310 & -.225 \\
\hline
\end{tabular}

En dicha tabla se aprecia que, en la muestra total de niños, se asociaron positivamente las variables del IMC con el esfuerzo bajo, la ansiedad generalizada, el miedo al daño físico, y el puntaje de la escala total de ansiedad con la comida chatarra.

En las niñas se halló una relación positiva entre la comida chatarra y la ansiedad generalizada, el miedo al daño físico y la fobia social, y que el esfuerzo alto (elegir sumas más difíciles) se asocia negativamente con ansiedad generalizada y fobia social. En los niños no se obtuvie- ron correlaciones significativas. Para el subgrupo de sobrepeso se obtuvo una correlación positiva entre la edad y la inmediatez. En el de obesidad se encontraron relaciones entre inmediatez y ansiedad por separación. En cuanto a los niños de 3ro. de primaria se halló una relación positiva entre inmediatez, IMC y ansiedad por separación, así como puntajes totales de ansiedad; en los de 4to. no se encontraron correlaciones significativas para dichas variables; mientras que en los de $5^{\circ}$, la comida chatarra se asoció positivamente con 
miedo al daño físico, pánico con agorafobia y ansiedad total. Además, la ansiedad por separación se asoció con la inmediatez, y negativamente con el IMC. El esfuerzo bajo lo hizo positivamente con el IMC, y negativamente con la ansiedad generalizada, y la fobia social con el esfuerzo alto (sumas difíciles). En cuanto a la agorafobia, se asoció negativamente con el programa de reforzamiento IV de 5 segundos de espera para contestar una suma.

En la escuela 1 se encontraron relaciones positivas entre la comida chatarra, la ansiedad generalizada, la ansiedad total, la ansiedad por separación y la inmediatez.
Finalmente, se llevó a cabo un análisis de regresión con procedimiento hacia adelante con filtro en el sexo (niña y niño), donde las variables de elección (Calidad, Inmediatez, Esfuerzo y Programa de reforzamiento) fueron dependientes de las subescalas de ansiedad (predictoras).

De acuerdo al análisis de regresión múltiple, usando el método por pasos hacia adelante y filtrando por sexo, se observa que las variables de elección se relacionan con las subescalas de ansiedad utilizando como criterio de significacncia el intervalo de confianza (límites inferior y superior). En las siguientes tablas se muestran los modelos obtenidos para cada dimensión de elección.

Tabla 3. Regresión lineal en el grupo de niñas.

\begin{tabular}{|c|c|c|c|c|c|c|}
\hline \multicolumn{7}{|c|}{ Niñas } \\
\hline Modelo & VD: Calidad & B & E.S. & $\mathbf{r}^{2}$ & L.I. & L.S. \\
\hline 1 & Miedo al daño físico & 0.719 & 0.339 & 0.074 & 0.040 & 1.397 \\
\hline \multirow{2}{*}{1} & \multicolumn{6}{|l|}{ VD: Esfuerzo } \\
\hline & Fobia social & -1.761 & 0.741 & 0.191 & -3.290 & -0.232 \\
\hline \multirow{2}{*}{2} & Fobia social & -1.964 & 0.670 & 0.191 & -3.350 & -0.577 \\
\hline & IMC & 2.489 & 0.960 & 0.374 & 0.503 & 4.475 \\
\hline \multirow{2}{*}{1} & \multicolumn{6}{|c|}{ VD: Programa de reforzamiento } \\
\hline & Trastorno obsesivo-compulsivo & -0.992 & 0.448 & 0.170 & -1.917 & -0.067 \\
\hline
\end{tabular}

Nota: VD: Variable dependiente, b: beta, E.S.: error estándar, L.I.: Límite inferior, L.S.: Límite superior, IMC: índice de masa corporal, $\mathrm{r}^{2}$ : coeficiente de determinación $\mathrm{r}^{2}$.

Como se puede observar en la Tabla 3, para la submuestra de niñas la Calidad es explicada significativamente por miedo al daño físico, y para la variable Esfuerzo el mejor modelo fue el que incluye la fobia social y el IMC, siendo esta última variable la que contribuye más al modelo; en el caso del Programa de reforzamiento, la única variable de explicación significativa fue el trastorno obsesivo-compulsivo. Para la variable Inmediatez
(Tabla 4) no se encontró ningún modelo significativo. Para la submuestra de niños, ningún modelo presentó una proporción de error reducido que resultara significativa. Además de lo anterior, fue de interés de este estudio distinguir a los participantes según su condición de sobrepeso u obesidad. Solamente un modelo resultó significativo para el grupo de obesidad: la Inmediatez explicada por la Ansiedad por separación.

Tabla 4. Regresión lineal para los niños con obesidad.

\begin{tabular}{|c|c|c|c|c|c|c|}
\cline { 3 - 7 } \multicolumn{2}{c|}{} & \multicolumn{5}{c|}{ Obesidad } \\
\hline Modelo & VD: Inmediatez & B & E.S. & $\mathbf{r}^{2}$ & L.I. & L.S. \\
\hline 1 & Ansiedad por separación & .376 & .148 & 0.182 & .073 & .678 \\
\hline
\end{tabular}

Nota: VD: Variable dependiente, b: beta, E.S.: error estándar, L.I.: Límite inferior, L.S., Límite superior, IMC: índice de masa corporal, $\mathrm{r}^{2}$ : coeficiente de determinación $\mathrm{r}^{2}$.

Es posible apreciar que la escala de ansiedad por separación reduce el nivel de error $\left(r^{2}=0.182\right)$ para la dimensión de Inmediatez en los niños con obesidad. 
Finalmente, se segmentó la muestra por grado, hallándose un modelo significativo que incluye Inmediatez como variable de respuesta y ansiedad por separación como variable predictora $\left(r^{2}=0.48\right)$ en los niños de tercer grado (Tabla 5).

Tabla 5. Regresión lineal para los niños de 3ro. y 5to. grados.

\begin{tabular}{|c|c|c|c|c|c|c|}
\hline Modelo & \multicolumn{6}{|c|}{ 3er. GRADO } \\
\hline \multirow{2}{*}{1} & VD: Inmediatez & B & E.S. & $\mathbf{r}^{2}$ & L.I. & L.S. \\
\hline & Ansiedad por separación & 0.704 & 0.203 & 0.48 & 0.265 & 1.143 \\
\hline Modelo & \multicolumn{6}{|c|}{ 5to. GRADO } \\
\hline \multirow{7}{*}{1} & VD: Calidad & B & E.S. & $\mathbf{r}^{2}$ & L.I. & L.S. \\
\hline & Pánico con agorafobia & 1.075 & 0.36 & 0.28 & 0.331 & 1.819 \\
\hline & VD: Inmediatez & B & E.S. & $\mathbf{r}^{2}$ & L.I. & L.S. \\
\hline & IMC & -1.760 & 0.6 & 0.27 & -3 & -0.520 \\
\hline & VD: Esfuerzo & B & E.S. & $\mathbf{r}^{2}$ & L.I. & L.S. \\
\hline & IMC & 3.098 & 0.86 & 0.36 & 1.329 & 4.867 \\
\hline & VD: Programa de reforzamiento & B & E.S. & $\mathbf{r}^{2}$ & L.I. & L.S. \\
\hline 1 & Pánico con agorafobia & -0.79 & 0.38 & 0.16 & -1.580 & 0 \\
\hline \multirow{2}{*}{2} & Pánico con agorafobia & -0.82 & 0.35 & 0.16 & -1.540 & -0.090 \\
\hline & Ansiedad por separación & -1.23 & 0.53 & 0.32 & -2.337 & -0.128 \\
\hline
\end{tabular}

Nota: VD: Variable dependiente, b: beta, E.S.: error estándar, L.I.: Límite inferior, L.S.: Límite superior, IMC: índice de masa corporal, $\mathrm{r}^{2}$ : coeficiente de determinación $\mathrm{r}^{2}$.

En el caso de los niños de quinto grado, los análisis de regresión múltiple muestran que la variable Calidad se asocia con la escala de Pánico con agorafobia; el IMC reduce el error de manera significativa cuando se evalúan Inmediatez y Esfuerzo como variables de respuesta $\left(r^{2}=0.27\right.$ y r $^{2}=0.36$, respectivamente). La variable Programa de reforzamiento se predice apropiadamente por Pánico con agorafobia y Ansiedad por separación.

Se observa en la variable de elección de comida chatarra que el miedo al daño físico explica $7.4 \%$. En cuanto al esfuerzo bajo (sumas fáciles), lo explican en $37 \%$ la fobia social en interacción con el IMC. Y finalmente la subescala de trastorno obsesivo-compulsivo explica en $17 \%$ elegir programas de reforzamiento altos (pocos segundos). Para el subgrupo de sobrepeso, la edad explica en $22 \%$ lo inmediato de los alimentos. En cuanto a los niños con obesidad, la subescala de ansiedad por separación explica la inmediatez en $14 \%$. En la escuela semiurbana, el trastorno obsesivo-compulsivo predice en 14\% el programa de reforzamiento, y en los niños de 3ro. se encontró que la inmediatez es explicada en $48 \%$ por la subescala de ansiedad por separación.
Finalmente, en los niños de 5to. grado se encontraron diversas interacciones entre la Calidad, explicada por el pánico con agorafobia en $28 \%$; la inmediatez por el IMC en $27 \%$; el esfuerzo por el IMC en $36 \%$; el programa de reforzamiento se explica en $32 \%$ por el pánico con agorafobia y la ansiedad por separación en conjunto.

Cabe mencionar que en los modelos que incorporaban al 4to. grado no se encontró relación alguna entre las variables analizadas.

\section{DISCUSIÓN}

El propósito de esta investigación fue observar la relación entre las dimensiones de impulsividad y las subescalas de ansiedad en niños con problemas de sobrepeso y obesidad, describir los puntajes promedio de las variables de ansiedad y las dimensiones de elección en niños con sobrepeso y obesidad a partir del género y el grado escolar, así como también identificar si los indicadores de ansiedad son predictores de las dimensiones de elección, y si estas predicciones difieren según la condición de salud (sobrepeso u obesidad), el sexo y el grado de escolaridad de los infantes. 
De manera general, los puntajes de ansiedad para los participantes de este trabajo se mantuvieron en un nivel moderado. Dichos resultados coinciden con los presentados por varios autores (Corbí y Pérez, 2011; Miranda y Gloria, 2018), quienes reportan que la ansiedad y la obesidad guardan una relación positiva, pero también niveles de ansiedad moderados - lo cual se asocia a la percepción errónea de que "los niños gorditos son más saludables" (Cabello y De Jesús 2011) -, ya que la tendencia a la normalización de los problemas de peso de los niños puede ser un factor que explique el desarrollo de niveles moderados de ansiedad, depresión y autopercepción corporal; no percibir como un problema la condición relacionada con el peso puede traducirse en la desatención de la obesidad, y esta percepción equívoca de la apariencia personal limita la efectividad de evaluaciones diagnósticas y tratamientos.

Los tutores principales (padre, madre o cuidador) son los que proveen y determinan la dieta común del menor. El aprendizaje a través de la observación y la imitación adquieren relevancia para este, formando así un patrón de comportamiento que se relacionará con sus elecciones de alimentos (Laessle, Uhl y Lindel, 2001), de modo que el comportamiento alimentario familiar influye en el desarrollo de la obesidad (Puder y Munsch, 2010).

De acuerdo con los resultados de los índices de correlación, que se observan en un nivel moderado en la mayoría de las asociaciones entre las subescalas de ansiedad (Ansiedad generalizada, Miedo al daño físico y Fobia social, TOC, Ansiedad por separación y Ansiedad total) y las dimensiones de elección de comida no saludable y opciones inmediatas, es posible suponer que tales variables participan en forma condicional en la elección de alimentos, lo que reafirma lo hallado por Nederkoorn et al. (2006) y Sánchez et al. (2013), quienes muestran un bajo control inhibitorio y una mayor sensibilidad de los niños con obesidad en la elección de comida no saludable.

En cuanto al análisis de regresión para determinar el nivel de predicción de las subescalas de ansiedad sobre las dimensiones asociadas a la elección, se encontró en general que, en el subgrupo de mujeres, la variación del IMC y la fobia social explicaron $37 \%$ de la varianza de la dimen- sión de esfuerzo en la variable Impulsividad; es decir, en las niñas con mayores puntajes en fobia social e IMC su elección depende en parte de lo difícil que sea una suma, de modo que regularmente eligieron las sumas fáciles. Este tipo de fobia se asocia al temor persistente de hablar en público, y regularmente quienes la sufren tienden a evitar los lugares donde haya multitudes, de acuerdo con lo señalado por Cebolla, Baños, Botella, Lurbe y Torró (2011) y Topçu, Orhon, Tayfun, Uçaktürk y Demirel (2016). En otras palabras, las mujeres harán una elección que les demande poco esfuerzo, lo que está asociado a un aumento en el peso y de la fobia social, lo que puede mantener su elección de comida no saludable porque les implica un menor esfuerzo conseguirla. Aunque los datos no son concluyentes, demuestran las posibles diferencias de género que deben ser consideradas en los programas de prevención.

En cuanto al subgrupo de sobrepeso, se obtuvieron resultados según la edad como predictor de la inmediatez con que eligen los niños una opción; en el grupo de obesos, la ansiedad por separación también predice la elección de opciones inmediatas, lo que explica $18 \%$ de la varianza. El paradigma de elección propuesto en el presente estudio, que incorpora una evaluación conductual para que a través de cuatro dimensiones de respuesta, y que toma en cuenta una variable psicológica como la ansiedad como predictora en niños con sobrepeso y obesidad, no aporta una evidencia contundente que describa tal relación, aunque algunos estudios preliminares encontraron que dichos niños tendían a efectuar una elección impulsiva, ya que preferían lo inmediato, con poco esfuerzo y seleccionaban regularmente la comida no saludable (Cavita y Sánchez, 2012; Romero y Zúñiga, 2016; Vite et al., 2020). En este sentido, la presente investigación aporta el análisis por subgrupos de una muestra de niños con sobrepeso y obesidad a fin de que las variables de grado sexo y condición sean incorporadas al análisis y a las estrategias de prevención.

Una de las variables más explicada por la ansiedad por separación fue la inmediatez, especialmente en el subgrupo de tercer grado, con $48 \%$ de varianza explicada de esta dimensión de la impulsividad. En México, en dicho grado los niños se 
hallan entre 7 y 8 años, edad en la que se desarrolla el proceso de toma de decisiones (Berger, 2014), que en el presente estudio se relaciona con la elección de alimentos con base en la cantidad, el tiempo de espera para recibirlos y el esfuerzo que se hará para obtenerlos (Neef et al., 2001, Neef et al., 2005). Como ya se mencionó, los padres determinan el tipo de dieta que lleva a cabo el niño, aunque fuera de casa los niños comienzan a hacer elecciones distintas, aunque basadas en el patrón de comportamiento alimenticio adquirido (Bandura, 1978; Fields et al., 2013; Giel et al., 2017; Goldschmidt et al., 2019); aun así, el acceso a alimentos no saludables, de acceso inmediato, ya no están reguladas por los adultos, por lo que un programa de promoción de hábitos saludables que incorpore variables de elección de alimentos puede ser de utilidad (Vite et al., 2020).

Respecto a los niños de quinto grado (niños de 10-11 años), los resultados de la regresión muestran que es el subgrupo que tiene diferentes modelos que explican las cuatro dimensiones del reforzador, la Calidad se explica por el pánico con agorafobia, la inmediatez y el esfuerzo por el IMC, y finalmente el tiempo de espera de una pantalla por la ansiedad por separación y el pánico con agorafobia. Lo anterior puede estar relacionado con el grado de madurez cerebral y la toma de decisiones en esta etapa previa a la preadolescencia (Logan, 1985; Rosselli, 2003). Por lo tanto, se propone incluir la madurez fisiológica como un factor intrínseco en la toma de decisiones (Berger, 2014).

En México no hay diferencias claras en cuanto al espectro de la ansiedad entre niñas y niños (Benjet et al., 2009; Cárdenas, Feria, Palacios y De la Peña, 2010) debido a que no se han llevado a cabo investigaciones en ese sentido, por lo que se considera pertinente el estudio epidemiológico de la ansiedad para tener mayor claridad del papel que desempeña en los trastornos alimenticios. En el presente estudio se obtuvieron puntajes promedio de ansiedad en un nivel moderado y con una alta variabilidad de los datos, lo que refleja la necesidad de aumentar el tamaño de la muestra y de tener un mayor control en cuanto a la equivalencia de los grupos. El estudio aporta asimismo una evaluación instrumental conductual que permite el estudio de los factores implicados en el problema de la obesidad infantil. Su aportación teórica se considera importante dado que la literatura en México sobre esta línea en particular es limitada. Algunos estudios muestran evaluaciones de impulsividad por medio de instrumentos psicométricos (Corbí et al., 2011: Guajardo, Ramírez y Guerrero, 2010; Guerrieri, Nederkoorn y Jansen, 2008; Lindberg, Hagman, Danielsson, Marcus y Persson, 2020) donde los datos presentados nos dan cuentra de un perfil general sobre lo que se describe como ansiedad. No obstante queda de soslayo el repertorio conductual en cuanto a la elección de opciones que son variables situacionalmente, y que reclama la identificación de patrón típico de elección (a partir de las regularidades que lo cararctericen).

Con el diseño adoptado para este estudio, también se aporta en relación al uso de instrumentos sensibles, que permiten abordar el espectro de la conducta impulsiva considerado un fenómeno multidimensional no estable; se vuelve una necesidad el uso de diferentes herramientas de medición para obtener una muestra más amplia del comportamiento (Neef et al., 2005), siendo la evaluación de la conducta impulsiva aquí propuesta, una alternativa de gran valor para el estudio de la eleccion de alimentos.

En este sentido la presente propuesta gira en torno a la instrumentación del establecimiento de un patrón conductual de elección con base a una toma de decisiones asociadas al contexto obesogénico, esto es mediante un software computacional sensible para la detección de comportamiento impulsivo bajo cuatro dimensiones de elección en cuanto a la comida, asociadas al tiempo que tardan en elegir un alimento, qué tanto esfuerzo les implica, así como la calidad del producto elegido.

En el estudio de la impulsividad al relacionarla con variables psicológicas como la ansiedad ha sido relevante para el complejo de distintas áreas en la salud física y psicológica, ya que los componentes de una intervención podrían considerarse seriamente en la implementación de estrategias que tomen en cuenta el papel de la impulsividad (Guajardo et al., 2010; Guerrieri et al., 2008; Romero et al., 2015). Esta forma de medición puede ser de mayor utilidad para identificar patrones 
conductuales que se asocian con el sobrepeso y la obesidad (Lindberg et al., 2020; Winstanley, Eagle y Robbins, 2006).

Para futuras investigaciones se recomienda ampliar el tamaño de la muestra, aumentar el control de las variables, disponer de un grupo control y de grupos equivalentes, incorporando a niños con normopeso para comparar los resultados. En este trabajo no fue posible obtener dicho grupo dado que los permisos otorgados por las instancias primarias participantes fueron otorgados para estudiar el comportamiento de niños con problemas de sobrepeso y obesidad, por lo que no se logró evaluar a niños sin esa condición.

Se propone, por último, incluir la variable de impulsividad en los programas de intervención, con su metodología de evaluación a lo largo de líneas base para obtener patrones de comportamiento y, en consecuencia, dirigir los esfuerzos para enseñar a los niños a elegir comida saludable, no inmediata y que requiera un mayor esfuerzo para obtenerla. En lo que respecta a la ansiedad, sería interesante desarrollar instrumentos válidos y confiables que incorporen variables conductuales como la impulsividad.

\section{AGRADECIMIENTOS}

Los autores agradecen al Consejo Nacional de Ciencia y Tecnología la beca otorgada (449445): Becario: 299266.

Citación: Alfaro B., J.N., Romero S., P. y Vite S., A. (2021). Ansiedad como predictor de la conducta impulsiva en niños con sobrepeso y obesidad. Psicología y Salud, 31(2), 179-194. https://doi.org/10.25009/pys.v31i2.2701.

\section{REFERENCIAS}

Bandura, A. (1978). Social learning theory of aggression. Journal of Communication, 28(3), 12-29.

Baumrind, D. (1971). Child care practices anteceding three patterns of preschool behavior. Genetic Psychology Monographs, 75(1), 43-88.

Benjet, C., Borges, G., Medina-Mora, M.E., Méndez, E., Fleiz, C., Rojas, E. y Cruz, C. (2009). Diferencias de sexo en la prevalencia y severidad de trastornos psiquiátricos en adolescentes de la Ciudad de México. Salud Mental, $32(2), 155-163$.

Berger, K.S. (2014). The developing person through the life span. New York: Worth Publishers.

Cabello G., M. y De Jesús R., D. (2011). Percepción de las madres de niños con obesidad sobre los hábitos alimenticios y sus responsabilidades en la alimentación de los hijos. Revista de Salud Pública y Nutrición, 12(1). Disponible en http://respyn. uanl.mx/index.php/respyn/article/view/281.

Caporal M., L., Moysen C., A. y Vite S., A. (2013, octubre). Desarrollo de autocontrol en niños con problemas de comportamiento. Memorias del XXI Congreso Mexicano de Psicología, Guadalajara, Jal., México.

Cárdenas E., M., Feria, M., Palacios, L. y De la Peña, F. (2010). Guía clínica para los trastornos de ansiedad en niños y adolescentes. México: Instituto Nacional de Psiquiatría Ramón de la Fuente y Secretaría de Salud.

Cavita C., C. y Sánchez S., A. (2012). Modelo de autocontrol basado en la ley de igualación en niños con sobrepeso y obesidad. Tesis inédita de licenciatura. México: UNAM.

Cebolla, A., Baños, R.M., Botella, C., Lurbe, E. y Torró, M.I. (2011). Perfil psicopatológico de niños con sobrepeso u obesidad en tratamiento de pérdida de peso. Revista de Psicopatología y Psicología Clínica, 16(2), 125-134.

Chamberlain, S.R., Derbyshire, K.L., Leppink, E. y Grant, J.E. (2015). Obesity and dissociable forms of impulsivity in young adults. CNS Spectrums, 20(5), 500-507.

Corbí, B. y Pérez N., M.A. (2011). Relación entre impulsividad y ansiedad en los adolescentes. Revista Electrónica de Motivación y Emoción, 37(14), 109-122.

Davis, C., Patte, K., Levitan, R., Reid, C., Tweed, S. y Curtis, C. (2007). From motivation to behaviour: A model of reward sensitivity, overeating, and food preferences in the risk profile for obesity. Appetite, 48, 12-19.

Fields, S.A., Sabet, M. y Reynolds, B. (2013). Dimensions of impulsive behavior in obese, overweight, and healthy-weight adolescents. Appetite, 70, 60-66. 
Giel, K.E., Teufel, M., Junne, F., Zipfel, S. y Schag, K. (2017). Food-related impulsivity in obesity and binge eating disorder -a systematic update of the evidence. Nutrients, 9(11), 1170. Recuperado de https://doi.org/10.3390/nu9111170.

Goldschmidt, A.B., Smith, K.E., Lavender, J.M., Engel, S.G. y Haedt-Matt, A. (2019). Trait-level facets of impulsivity and momentary, naturalistic eating behavior in children and adolescents with overweight/obesity. Journal of Psychiatric Research, $110,24-30$.

Gran, B.C. y Nieto, M.A.P. (2012). Impacto de la impulsividad en el consumo de alcohol, adolescente. EduPsykhé: Revista de Psicología y Psicopedagogía, 11(1), 21-32.

Guajardo E., G.P., Ramírez M., T.G. y Guerrero F., T. (2010). Ansiedad y depresión en niños con sobrepeso y obesidad: Resultados de un campo de verano. Summa Psicológica UST, 7(2), 67-74.

Guerrieri, R., Nederkoorn, C. y Jansen, A. (2008). The effect of an impulsive personality on overeating and obesity: Current state of affairs. Psihologijske Teme, 17(2), 265-286.

Gutiérrez J., D.S., Valladolid G., R. y De Fonseca F., R. (2013). La impulsividad: ¿antesala de las adicciones comportamentales? Health and Addictions, 13(2), 145-155.

Hernández, G., Minor, E. y Aranda, R. (2012). Determinantes económicos: Evolución del costo de las calorías en México. En J. Á. Rivera-Dommarco, C. A. Aguilar S. y M. Hernández Á. (Eds.): Obesidad en México: Recomendaciones para una política de Estado (pp. 145-164). México: UNAM.

Hernández G., L., Bermúdez O., G., Spence, S.H., González M., M., Martínez G., J., Aguilar V., J. y Gallegos G., J. (2010). Versión en español de la Escala de Ansiedad para Niños de Spence (SCAS). Revista Latinoamericana de Psicología, 42(1), 13-24.

Iribarren M., M., Jiménez G., M., García de C., J.M. y Rubio V., G. (2011). Validación y propiedades psicométricas de la Escala de Impulsividad Estado (EIE). Actas Española de Psiquiatría, 39(1), 49-60.

Laessle, R.G., Uhl, H. y Lindel, B. (2001). Parental influences on eating behavior in obese and nonobese preadolescents. International Journal of Eating Disorders, 30(4), 447-453.

Lindberg, L., Hagman, E., Danielsson, P., Marcus, C. y Persson, M. (2020). Anxiety and depression in children and adolescents with obesity: a nationwide study in Sweden. BMC Medicine, 18(1), 1-9.

Logan, G.D. (1985). Executive control of thought and action. Acta Psychologica, 60(2-3), 193-210.

Luna H., J., Ramírez D., M., Guerrero C., I., Guevara S., R., Marín V., J. y Jiménez A., E. (2020). Evaluación del estado nutricional de niños en edad escolar de dos localidades indígenas de Oaxaca. Revista de Salud Pública y Nutrición, 19(1), 1-9.

Márquez C., M.E., Arroyo G., E., Granados R., A. y Ángeles L., A. (2017). Hospital Psiquiátrico Infantil Dr. Juan N. Navarro: 50 años de atención a la salud mental de niños y adolescentes en México. Salud Pública de México, 59(4), 477-484.

Meule, A. (2013). Impulsivity and overeating: a closer look at the subscales of the Barratt Impulsiveness Scale. Frontiers in Psychology, 4, 177. Recuperado de https://doi.org/10.3389/fpsyg.2013.00177.

Miranda, O. y Gloria, E. (2018). Alteraciones psicológicas asociadas a la obesidad infantil. Revista Medica Herediana, 29(2), 111-115.

Nederkoorn, C., Braet, C., Van Eijs, Y., Tanghe, A. y Jansen, A. (2006). Why obese children cannot resist food: the role of impulsivity. Eating Behaviors, 7(4), 315-322.

Nederkoorn, C., Coelho, J.S., Guerrieri, R., Houben, K. y Jansen, A. (2012). Specificity of the failure to inhibit responses in overweight children. Appetite, 59(2), 409-413.

Neef, N.A., Bicard, D.F. y Endo, S. (2001). Assessment of impulsivity and the development of self-control in students with attention deficit hyperactivity disorder. Journal of Applied Behavior Analysis, 34(4), 397-408.

Neef, N.A. y Lutz, M.N. (2001). A brief computer-based assessment of reinforcer dimensions affecting choice. Journal of Applied Behavior Analysis, 34(1), 57-60. doi: 11317987.

Neef, N.A., Mace, F.C. y Shade, D. (1993). Impulsivity in students with serious emotional disturbance: The interactive effects of reinforcer rate, delay, and quality. Journal of Applied Behavior Analysis, 26(1), 37-52.

Neef, N.A., Marckel, J., Ferreri, S.J., Bicard, D.F., Endo, S., Aman, M.G. y Armstrong, N. (2005). Behavioral assessment of impulsivity: A comparison of children with and without attention deficit hyperactivity disorder. Journal of Applied Behavior Analysis, 38(1), 23-37.

Negrete C., A., Cuevas, E. y Vite S., A. (2009). Sistema de evaluación de la conducta impulsiva (SECI). México: UNAM y Hospital General Ss.

Negrete C., A. y Vite S., A. (2011). Relación de la violencia familiar y la impulsividad en una muestra de adolescentes mexicanos. Acta Colombiana de Psicología, 14(2), 121-128.

Organización de las Naciones Unidas para la Alimentación y la Agricultura (2018). El estado mundial de la agricultura y la alimentación. Migración, agricultura y desarrollo rural. Roma: FAO.

Organización para la Cooperación y Desarrollo Económicos (2017). Obesidad y la economías de la prevención. París: OCDE. 
Organization for Economic Co-operation and Development. (2017). Obesity update 2017. París: OECD.

Organización Mundial de la Salud (2018). Datos y cifras sobre obesidad infantil. Ginebra: OMS.

Organización Mundial de la Salud (2019). Datos y cifras sobre obesidad infantil. Ginebra: OMS.

Pascual N., D., Pascual N., T., Redondo D., M. y Pérez N., M. (2014). Sensibilidad a la recompensa y al castigo, personalidad, impulsividad y aprendizaje: un estudio en un contexto de violencia de pareja. Clínica y Salud, 25(3), 167-174.

Patterson, G.R. y Wells, K.C. (1982). A social learning approach. En D. H. Barlow (Ed.): Coercive family process (v. 3). Eugene, OR: Castalia.

Pereira, C. y Chehter, E.Z. (2011). Associações entre impulsividade, compulsão alimentar e obesidade em adolescentes. Arquivos Brasileiros de Psicologia, 63(3), 16-30.

Plutchik, R., Van Praag, H.M., Conte, H.R. y Picard, S. (1989). Correlates of suicide and violence risk 1: The suicide risk measure. Comprehensive Psychiatry, 30(4), 296-302.

Puder, J.J. y Munsch, S. (2010). Psychological correlates of childhood obesity. International Journal of Obesity, 34(2), S37-S43.

Rivera D., J.A., Colchero, M.A., Fuentes, M.L., González de C., Martínez, T., Aguilar S., C.A., Hernández L., G. y Barquera, S. (2018). La obesidad en México. Estado de la política pública y recomendaciones para su prevención y control. México: INSP.

Romero B., T. y Zuñiga C., G. (2016). Relación entre ansiedad y conducta impulsiva en una muestra de niños escolares con sobrepeso. Tesis inédita de licenciatura. México: UNAM.

Romero P., E.M., De Paz F., J.A., Camberos C., N.A., Tánori T., J.M., Bernal R., F. y Márquez R., S. (2015). Evaluación de los estados de ansiedad y depresión de niños obesos después de participar en un programa de ejercicio físico. Biotecnia, 17, $11-15$.

Rosselli, M. (2003). Maduración cerebral y desarrollo cognoscitivo. Revista Latinoamericana de Ciencias Sociales, Niñez y Juventud, 1(1), 125-144.

Ryden, A., Sullivan, M., Torgerson, J.S., Karlsson, J., Lindroos, A.K. y Taft, C. (2003). Severe obesity and personality: A comparative controlled study of personality traits. International Journal of Obesity, 27, 1534-1540.

Salvo, L. y Castro, A. (2013). Confiabilidad y validez de la escala de impulsividad de Barratt (BIS-11) en adolescentes. Revista Chilena de Neuro-Psiquiatría, 51(4), 245-254.

Sánchez S., P., Giraldo H., J.J. y Quiroz P., M.F. (2013). Impulsividad: una visión desde la neurociencia del comportamiento y la psicología del desarrollo. Avances en Psicología Latinoamericana, 31(1), 241-251.

Shamah L., T. y Rivera D., J. (2020). Encuesta Nacional de Salud y Nutrición 2018 Resultados en localidades con menos de 100 000 habitantes. Salud Pública de México, 61(6), 707-708.

Sociedad Mexicana de Psicología (2010). Código ético del psicólogo. México: Trillas.

Topçu, S., Orhon, F. Ş., Tayfun, M., Uçaktürk, S. A. y Demirel, F. (2016). Anxiety, depression and self-esteem levels in obese children: a case-control study. Journal of Pediatric Endocrinology and Metabolism, 29(3), 357-361.

Torres, F. y Rojas, A. (2018). Obesidad y salud pública en México: transformación del patrón hegemónico de oferta-demanda de alimentos. Problemas del Desarrollo, 49(193), 145-169.

Torres M., V., Rodríguez H., C. y Silva J., L.C. (2020). Formas de malnutrición regional en México en el marco de un desarrollo sostenible. Estudios Sociales: Revista de Alimentación Contemporánea y Desarrollo Regional, 30(55), 7.

Vite S., A., Negrete C., A. y Cavita C., C. (2020). Promoting self-control in overweight and obese children. Nutrición Hospitalaria, 37(2), 251-259.

Winstanley, C.A., Eagle, D.M. y Robbins, T.W. (2006). Behavioral models of impulsivity in relation to ADHD: Translation between clinical and preclinical studies. Clinical Psychology Review, 26(4), 379-395.

World Health Organization (2013). Population-based approaches to childhood obesity prevention. Geneve: WHO.

World Health Organization (2018). Obesity and overweight. Geneve: WHO.

World Health Organization (2019). Adolescent mental health. Fact sheets. Geneve: WHO.

Instituto de Investigaciones Psicológicas - Universidad Veracruzana

ISSN impreso: 1405-1109
Psicología y Salud, Vol. 31, Núm. 2: 179-194, julio-diciembre de 2021

https://doi.org/10.25009/pys.v31i2.2701 